\title{
Strengths, weaknesses, and applications of computational axial lithography in tissue engineering
}

\author{
Comments on B. E. Kelly et al., Volumetric additive manufacturing via tomographic \\ reconstruction. Science. 363, 1075-1079 (2019)
}

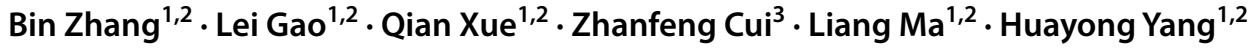

Published online: 29 January 2020

(c) Zhejiang University Press 2020

Recently, Kelly and colleagues [1], inspired by computed tomography (CT), report a "volumetric additive manufacturing" technology via a computed axial lithography (CAL) approach. A related US patent application [2] has also been filed. The cumulative light exposure solidifies the material in the target area, while the other area remains uncured, resulting in only specific points in the designed 3D objects being printed. This technology significantly improves the capability of the digital light processing (DLP) technique. Meanwhile, the lithography approach based on a similar algorithm was already proposed by Xiang $\mathrm{Wu}$ in a patent (application No. PCT/CN2016/080097) in 2016 [3]. Although this approach can theoretically achieve high resolutions, similar issues to conventional DLP approaches remain in the following aspects:

(1) Its compatibility is limited to photosensitive materials, thus restricting its capability in fabricating multimaterial or microstructural constructs. For example, the solidified area is suspended in liquid during the curing process. High-viscous or solid precursor materials were then added to reduce the displacement and the geometric dislocation-induced blurring. However, this may

Liang Ma

liangma@zju.edu.cn

Huayong Yang

yhy@zju.edu.cn

1 State Key Laboratory of Fluid Power and Mechatronic Systems, Zhejiang University, Hangzhou 310058, China

2 School of Mechanical Engineering, Zhejiang University, Hangzhou 310058, China

3 Department of Engineering Science, Institute of Biomedical Engineering, University of Oxford, Oxford OX3 7DQ, UK result in residues of the precursor material remaining in the structure.

(2) The effects of the attenuating oxygen content and the diffusion of the oxygen/inhibiting molecules on the accuracy of this technology need further investigation. An oxygen inhibition method has been proposed to delay the curing time. By dissolving oxygen or other inhibiting molecules sufficiently in the printing material, the free radicals generated by the photoinitiator react preferentially with the inhibitor so that sufficient light intensity accumulates in the target area to ensure curing. However, the oxygen content of the liquid in the target position attenuates during the printing process, so the influence of the nonlinear attenuation on the material response needs further study. The authors suggested that the reaction of photoinitiator and oxygen required the most time in this CAL process. In the final stage of the reaction, the oxygen in the target position is lower than the threshold and the materials solidified rapidly. In this process, the diffusion oxygen and other inhibiting molecules inside this liquid-solid transform will require substantial additional research to improve fabrication accuracy.

(3) The scattering of light and the optical superposition influence manufacturing accuracy. The band of incident light is limited by adding dye and blurring caused by the refraction of light is reduced by the vertical incidence. However, due to the physical properties of the wall of the container and the interface between liquid and solid, incident light will inevitably lead to a certain degree of refraction, reflection, and attenuation. These changes in the optical path were neglected because the depth of focus of the projection system is considerably larger than the diameter of the printing volume. As the molecules aggregate, the solid-liquid interface also 
causes changes in the optical path, which may lead to energy loss and imaging errors. Therefore, these factors must be considered in the future to improve the printing accuracy.

Although there is controversy over CAL technology, with improvements in computing algorithms and the analysis of light, this technology will provide a technical breakthrough, especially in the areas of 3D bioprinting and regenerative medicine. We have been carrying out studies on 3D printing of cornea, skin, and cardiac patches [4], aiming to develop transplantable tissue grafts for the treatment of diseases and injuries, e.g., corneal blindness, skin burns, and myocardial infarction. As one of the initiators of the journal of BioDesign and Manufacturing, we are tracking the development of a variety of bio-manufacturing technologies. We believe that CAL has at least the following advantages over conventional additive manufacturing technologies. First, CAL has intrinsic advantages in fabricating tissues or organs in liquid materials. Currently, at least 15 types of tissues or organs can be 3D-printed using viscous liquid materials (bone, cartilage, cornea, nerve, muscle, blood vessel, lymphatic tissue, endocrine gland, uterus, ovary, cervicovaginal tissue, lung, airway, liver, kidney) [5, 6], three of which (cornea, liver, blood vessel) use DLP technology [7-9]. Second, CAL permits the construction of solid and smooth surfaces, enabling the fabrication of most tissues and organs. For example, a transparent cornea with a smooth surface can be fabricated and potentially transplanted to animals and even human patients. Finally, CAL has achieved unprecedented fabricating speed over all other 3D printing techniques, significantly accelerating the process from bench to bedside.

The volumetric additive manufacturing technique using this CAL technique is a major advancement in additive manufacturing in providing unprecedented fabricating speed and resolution. Once adapted to meet the requirements of biomedical applications, this technology will provide a revolutionary tool in tissue engineering and regenerative medicine.
Acknowledgements We would like to thank the support by Key Research and Development Projects of Zhejiang Province (Grant No. 2017C01054), National Key Research and Development Program of China (2018YFA0703000), Natural Science Foundation of China (Grant Nos. 51875518, 51821093), and the Fundamental Research Funds for the Central Universities (Grant Nos. 2019XZZX003-02, 2019FZA4002).

Conflict of interest Authors declare no conflict of interest.

\section{References}

1. Kelly BE, Bhattacharya I, Heidari H, Shusteff M, Spadaccini CM, Taylor HK (2019) Volumetric additive manufacturing via tomographic reconstruction. Science 363:1075-1079

2. Kelly B, Panas R, Shusteff M, Spadaccini C, Taylor H, Bhattacharya I (2018) System and method for computed axial lithography (CAL) for 3D additive manufacturing. https://patents.google.com/ patent/WO2018208378A2/en?oq=WO2018208378. Accessed 27 Mar 2019

3. Wu X (2016) Imaging and forming method using projection operation and back projection method. https://patents.google.com/ patent/WO2016173474A1/fi. Accessed 25 Mar 2019

4. Gao L, Zhang B, Ma L, Luo Y, Xue Q, Cui Z, Yang H (2018) Research lab on 3D bioprinting of Zhejiang University. Bio-Des Manuf 1:211-214

5. Vijayavenkataraman S, Yan W-C, Lu WF, Wang C-H, Fuh JYH (2018) 3D bioprinting of tissues and organs for regenerative medicine. Adv Drug Deliv Rev 132:296-332

6. Zhang B, Luo Y, Ma L, Gao L, Li Y, Xue Q, Yang H, Cui Z (2018) 3D bioprinting: an emerging technology full of opportunities and challenges. Bio-Des Manuf 1:2-13

7. Ma X, Qu X, Zhu W, et al (2016) Deterministically patterned biomimetic human iPSC-derived hepatic model via rapid 3D bioprinting. Proc Natl Acad Sci 113:2206-2211

8. Zhu W, Qu X, Zhu J, et al (2017) Direct 3D bioprinting of prevascularized tissue constructs with complex microarchitecture. Biomaterials 124:106-115

9. Warner J, Soman P, Zhu W, Tom M, Chen S (2016) Design and $3 \mathrm{D}$ printing of hydrogel scaffolds with fractal geometries. ACS Biomater Sci Eng 2:1763-1770 